\title{
DOUBLE-PEAKED NARROW-LINE ACTIVE GALACTIC NUCLEI. II. THE CASE OF EQUAL PEAKS
}

\author{
K. L. Smith ${ }^{1}$, G. A. Shields ${ }^{1}$, S. Salviander ${ }^{1,2}$, A. C. Stevensis ${ }^{1}$, and D. J. Rosario ${ }^{3}$ \\ ${ }^{1}$ Department of Astronomy, University of Texas, Austin, TX 78712, USA; klsmith@astro.umd.edu, shields@astro.as.utexas.edu, \\ triples@astro.as.utexas.edu, acs0196@mail.utexas.edu \\ 2 Department of Physics, Southwestern University, Georgetown, TX 78626, USA \\ ${ }^{3}$ Max-Planck-Institute for Extraterrestrial Physics, D-85748 Garching, Germany; rosario@mpe.mpg.de \\ Received 2011 August 1; accepted 2012 April 12; published 2012 May 25
}

\begin{abstract}
Active galactic nuclei (AGNs) with double-peaked narrow lines (DPAGNs) may be caused by kiloparsec-scale binary AGNs, bipolar outflows, or rotating gaseous disks. We examine the class of DPAGNs in which the two narrow-line components have closely similar intensity as being especially likely to involve disks or jets. Two spectroscopic indicators support this likelihood. For DPAGNs from Smith et al., the "equal-peaked" objects (EPAGNs) have $[\mathrm{Ne} \mathrm{v}] /[\mathrm{O}$ III] ratios lower than for a control sample of non-double-peaked AGNs. This is unexpected for a pair of normal AGNs in a galactic merger, but may be consistent with [O III] emission from a rotating ring with relatively little gas at small radii. Also, $[\mathrm{O} \mathrm{III}] / \mathrm{H} \beta$ ratios of the redshifted and blueshifted systems in the EPAGN are more similar to each other than in a control sample, suggestive of a single ionizing source and inconsistent with the binary interpretation.
\end{abstract}

Key words: galaxies: active - quasars: general

Online-only material: color figure

\section{INTRODUCTION}

Dual active galactic nuclei (AGNs) have recently attracted interest as examples of black hole fueling and AGN visibility during galaxy mergers. Several recent studies have considered objects with double-peaked narrow emission lines, particularly the [O III] $] \lambda 5007,4959$ line, as candidates for kiloparsec-scale binary AGNs (Zhou et al. 2004; Gerke et al. 2007; Comerford et al. 2009; Wang et al. 2009; Liu et al. 2010b; Smith et al. 2010). Two adjacent narrow-line regions (NLRs) associated with individual supermassive black holes (SMBHs) would result in a double-peaked profile for plausible line-of-sight orbital velocities. Recent imaging of double-peaked narrow-line AGNs (hereafter DPAGNs) has resulted in the discovery of a number of true binaries. Liu et al. (2010a) report four such objects, with dual active nuclei in optical slit spectra spatially coinciding with NIR-detected double stellar components. Also, Fu et al. (2011) report that 8 out of 17 observed Type 1 DPAGNs and 8 out of 33 observed Type 2 DPAGNs were found to be in mergers, although not necessarily in mergers where the orbital velocity between the galaxies is responsible for the observed line splitting. Shen et al. (2011) present five DPAGNs imaged to be kiloparsec-scale binaries, and McGurk et al. (2011) have reported a confirmed dual AGN in J0952+2552. Comerford et al. (2011) report a dual AGN selected by double-peaked [O III] as a Chandra double $\mathrm{X}$-ray source.

Double-peaked narrow lines can also result from biconical outflow, as in Mrk 78 (Whittle \& Wilson 2004) and in J1517+33 and J1129+60 (Rosario et al. 2010), or from a rotating gaseous disk. Shen et al. (2011) maintain that the majority (up to 90\%) of double-peaked [O III] objects are caused by NLR kinematics (i.e., jets and disks) instead of kiloparsec-scale binaries. Fu et al. (2012) find only 4 binary AGNs among 42 DPAGNs. A recent study by Tingay \& Wayth (2011) sought binary AGNs by looking for dual compact radio sources in 11 of the doublepeaked [O III] objects published by Wang et al. (2009). These authors find compact radio emission in only two of their sources, with neither detection being a dual source. In a recent theoretical study, Blecha et al. (2012) examine narrow-line profiles in galaxy mergers with fueling of the two central black holes. They find that double-peaked profiles often result from gas dynamics distinct from binarity of the active nuclei, but that double-peaked [O III] lines still represent a useful search method for finding dual AGNs. It is important to develop diagnostic tools to identify the likely cause of double-peaked narrow lines in a given AGN, in order to improve efficiency of finding dual AGNs as well as to promote understanding of the range of geometries that occur in the NLR of AGNs.

In this paper, we focus on DPAGNs in which the two components of the narrow lines have substantially equal strength ("equal-peaked AGNs" or EPAGNs). This is motivated in particular by the existence of a subset of DPAGNs with strikingly symmetrical profile, which bring to mind the possibility of a rotating ring or disk as the cause of the double peak. Such objects might also represent biconical outflows, but they seem unlikely to be dual AGNs, for which there is no reason to expect equal component fluxes. In Section 2, we describe how these objects were selected from the larger sample of DPAGNs. In Section 3, we look at the high-ionization line ratios of the EPAGNs and compare the line ratios in the redshifted and blueshifted systems. We find that these properties of the EPAGNs as a group are inconsistent with a binary interpretation. The properties we find are consistent with a rotating-disk interpretation for the doublepeaked [O III] profile, but we stress that this interpretation is not unique. Our conclusions are summarized in Section 4. We assume a cosmology with $\Omega_{\Lambda}=0.7, \Omega_{m}=0.3$, and $H_{0}=70 \mathrm{kms}^{-1} \mathrm{Mpc}^{-1}$.

\section{SELECTION OF EQUAL-PEAKED OBJECTS}

The original selection of the double-peaked [O III] objects is described in Section 4 of Smith et al. (2010). The objects were selected from SDSS DR7, which contains 21,592 QSO spectra in the redshift range of $0.1 \leqslant z \leqslant 0.7$. This keeps the [O III] $] \lambda \lambda 5007,4959$ line in the Sloan Digital Sky Survey (SDSS) spectral window and out of the water vapor forest. 


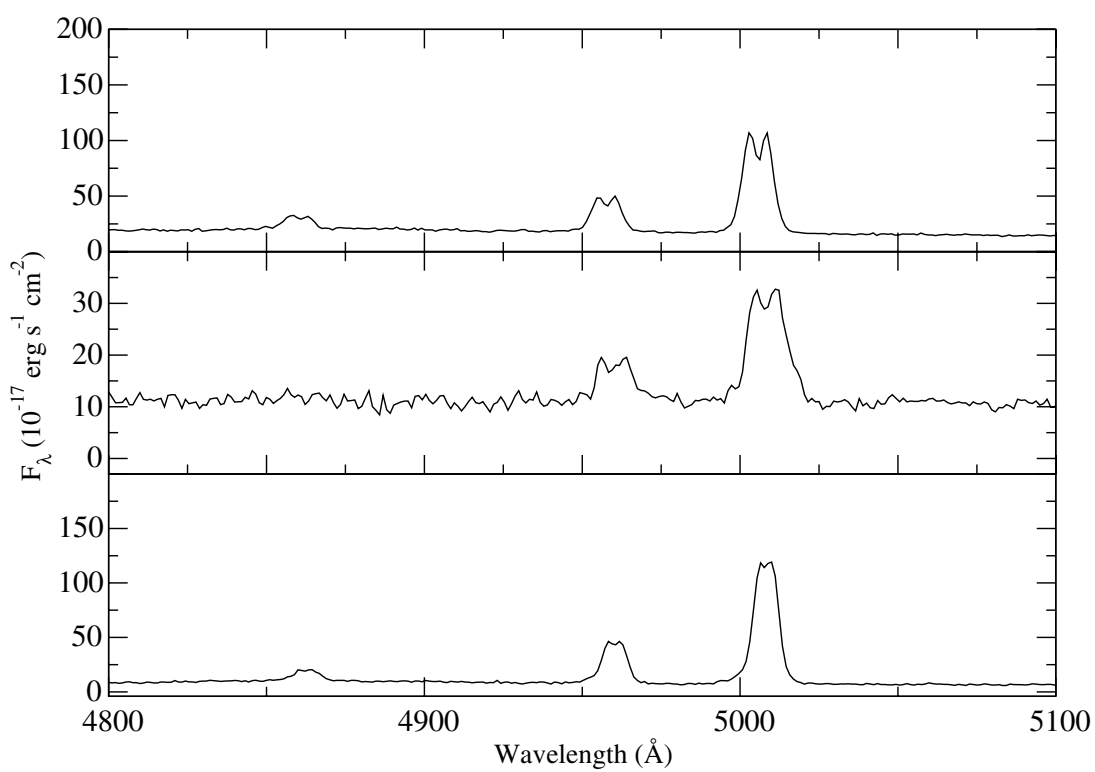

Figure 1. Three spectra typical of the eye-selected equal-peaked objects (EHAGNs). Note the similarity in height and structure of the two components of $[\mathrm{O}$ III $] \lambda \lambda 5007,4959$.

Table 1

Equal-Height Double-peaked AGNs

\begin{tabular}{lcllc}
\hline \hline SDSS Name & $\begin{array}{c}\text { Velocity Splitting } \\
\left(\mathrm{km} \mathrm{s}^{-1}\right)\end{array}$ & $z_{\text {SDSS }}$ & $F_{\mathrm{r}} / F_{\mathrm{b}}$ & Seyfert Type \\
\hline J101241.20+215556.0 & 210 & 0.111 & 1.027 & 1 \\
J113105.07+610405.1 & 330 & 0.338 & & 1 \\
J131018.47+250329.5 & 180 & 0.313 & 0.723 & 1 \\
J144105.64+180507.9 & 280 & 0.107 & & 1 \\
J151518.29+551535.3 & 250 & 0.513 & 1.214 & 1 \\
J153231.80+420342.7 & 300 & 0.21 & 0.878 & 1 \\
J082857.99+074255.7 & 300 & 0.554 & 1.464 & 2 \\
J123605.45-014119.1 & 330 & 0.211 & 1.012 & 2 \\
J124928.36+353926.8 & 380 & 0.527 & & 2 \\
J133226.34+060627.3 & 420 & 0.207 & 2.664 & 2 \\
J134415.75+331719.1 & 730 & 0.686 & 1.041 & 2 \\
J144157.24+094859.1 & 810 & 0.22 & & 2 \\
J171544.02+600835.4 & 350 & 0.157 & 0.654 & 2 \\
\hline
\end{tabular}

Notes. Objects with equal-height components in the double-peaked [O III] line selected by eye (EHAGNs), as described in Section 2. Note that the ratio of the fluxes in these objects is not necessarily near unity. Objects with equal peaks selected by flux ratio are given in Table 2 .

A visual inspection found 148 DPAGNs, of which 62 were Type 2 and 86 Type 1 , with an average redshift of 0.33 .

We used two different methods to identify the equal-peaked objects. The first method is selection by eye. Symmetrical appearance was the basis for this selection, i.e., the red and blue components are of similar width and height. Several of these objects are "dimples," where the components are separated by only a small depression at the top of the line profile. (It is these that we most suspect of being rotating disks.) Others are more widely spaced and have a deeper gap between the peaks, but otherwise are similar in shape. Examples are shown in Figure 1. We identified 13 such objects out of the 148 total DPAGNs. Henceforth, objects selected in this fashion are referred to as "equal-height AGNs" or EHAGNs. The objects thus selected are listed Table 1; there are 6 EHAGNs of Type 1 and 7 of Type 2.

A more quantitative way to identify equal-peaked objects is by using the ratio of the component fluxes. In Table 3 of Smith et al. (2010), we reported the component fluxes for 78 objects
Table 2

Equal-Flux Double-peaked AGNs

\begin{tabular}{lcccc}
\hline \hline SDSS Name & $\begin{array}{c}\text { Velocity Splitting } \\
\left(\mathrm{km} \mathrm{s}^{-1}\right)\end{array}$ & $z_{\text {SDSS }}$ & $F_{r} / F_{b}$ & Seyfert Type \\
\hline J081542.53+063522.9 & 280 & 0.244 & 0.8793 & 1 \\
J090615.92+121845.6 & 330 & 0.644 & 1.2467 & 1 \\
J091649.41+000031.5 & 310 & 0.222 & 0.8054 & 1 \\
J101241.20+215556.0 & 210 & 0.111 & 1.0270 & 1 \\
J120343.22+283557.8 & 850 & 0.374 & 0.8585 & 1 \\
J121911.16+042905.9 & 480 & 0.555 & 0.7735 & 1 \\
J124813.82+362423.6 & 350 & 0.207 & 0.8273 & 1 \\
J130724.08+460400.9 & 580 & 0.353 & 0.9400 & 1 \\
J133455.24+612042.1 & 215 & 0.495 & 0.9733 & 1 \\
J145110.04+490813.5 & 220 & 0.156 & 0.8031 & 1 \\
J145408.36+240521.3 & 300 & 0.535 & 0.7533 & 1 \\
J151518.29+551535.3 & 250 & 0.513 & 1.2140 & 1 \\
J153231.80+420342.7 & 300 & 0.210 & 0.8775 & 1 \\
J153423.19+540809.0 & 290 & 0.215 & 0.7596 & 1 \\
J084049.46+272704.7 & 580 & 0.136 & 0.8297 & 2 \\
J120526.04+321314.6 & 670 & 0.485 & 0.7785 & 2 \\
J123605.45-014119.1 & 330 & 0.211 & 1.0123 & 2 \\
J134415.75+331719.1 & 730 & 0.686 & 1.0410 & 2 \\
J140500.14+073014.1 & 340 & 0.135 & 0.8112 & 2 \\
J140816.02+015528.3 & 350 & 0.166 & 0.9778 & 2 \\
J151842.95+244026.0 & 280 & 0.561 & 1.0098 & 2 \\
J210449.13-000919.1 & 380 & 0.135 & 0.9397 & 2 \\
\hline
\end{tabular}

Note. Objects with equal-flux components in the double-peaked [O III] line (EFAGNs), as described in Section 2.

for which we obtained reliable double Gaussian fits. The fitting procedure assumed a Gaussian for each of the two components of the $\lambda 5007$ and $\lambda 4959$ lines, with adjustable velocity, FWHM, and flux, while maintaining the $3: 1$ flux ratio required by atomic physics. From these fluxes, we construct the ratio $F_{r} / F_{b}$, where $F_{r}$ and $F_{b}$ are the fluxes of the red and blue components, respectively. We take objects with $0.75 \leqslant F_{r} / F_{b} \leqslant 1.25$ to be "equal" and refer to them as "equal-flux AGNs" or EFAGNs. There are 13 EFAGNs AGN of Type 1 and 9 of Type 2, as listed in Table 2. Note that these lists are not mutually exclusive. There are 3 Type 1 and 2 Type 2 objects in common. 
Some of the EHAGNs did not have Gaussian fits we considered to be unique. Also, several of the EFAGNs were unequal to the eye and seem unlikely to be caused by a rotating disk unless it is eccentric or subject to asymmetrical obscuration. For these reasons, we analyze the EHAGNs and EPAGNs separately, and emphasize the former.

Shen et al. (2011) describe imaging and spatially resolved spectroscopy of a sample of DPAGNs from SDSS. Many of the objects are argued to be single AGNs with complex NLR kinematics, based on an absence of dual stellar nuclei and the nature of the $[\mathrm{O} \mathrm{III}]$ velocity field. These authors identify 10 double-peaked [O III] objects as likely to be caused by rotating gaseous disks. Five of these objects would have been classified as EHAGNs in our approach. The remaining five objects appear to be EFAGNs, with one exception, J0851+1327, whose [O III] line exhibits only a slight blue shoulder. Additionally, one of our EHAGNs and three of our EFAGNs appear in the imaged sample of Fu et al. (2012), where the authors conclude from high-resolution imaging and spectroscopy that the double-peaked [O III] results from NLR kinematics. Therefore, examples already exist in which EPAGNs have been determined to be caused by NLR kinematics, likely rotating disks, instead of binarity.

\section{THE NATURE OF THE EPAGNs}

Here, we consider two spectroscopic tests of the nature of the EPAGNs.

\subsection{High-ionization Lines}

One possible test of the nature of the EPAGNs involves the high-ionization narrow lines. It is widely believed that much of the emission in high-ionization lines such as [Ne v] and [Fe VII] comes from the core of the NLR, because they often have larger line widths and because they have weaker intensities in Type 2 AGNs (where the NLR core may be obscured). In the case of a rotating ring, the emission from such a core might be relatively weak. Thus, weakness of [ $\mathrm{Nev}]$ in the EPAGNs would be a point of consistency with a rotating disk geometry, although this would not be a unique interpretation. Such weakness would in any case argue against a dual AGN model, which presumably would involve two normal NLRs.

We examined the [Ne v] intensity of the EPAGNs, restricting attention to the Type 1 objects for which the NLR core should be visible. For the EHAGN, there are six objects (see Table 1), of which four have redshifts large enough for [ $\mathrm{Ne} \mathrm{v}]$ to be in the spectral window. For these, line fluxes were measured using the IRAF routine SPLOT. ${ }^{4}$ For $\mathrm{J} 1131+61$, [O III] is weak and $[\mathrm{Ne} \mathrm{v}]$ undetected. For the remaining three objects, the measured fluxes for [ $\mathrm{Ne} \mathrm{V}]$ and [O III] are given in Table 3. We estimate an uncertainty of $\pm 10 \%$ in the individual measurements of the flux in $[\mathrm{Ne} \mathrm{v}]$, based on the highest and lowest plausible continuum levels. The results give $I(\lambda 3426) / I(\lambda 5007)$ in the range $0.056-0.075$, for an average of $0.064 \pm 0.005$ (standard error of mean).

For comparison, the $[\mathrm{Ne} \mathrm{v}] /[\mathrm{O} \mathrm{III}]$ ratio in the overall SDSS Type 1 AGN composite by Vanden Berk et al. (2001) is 0.21. We made our own composite spectrum for a control sample of all SDSS AGNs in the redshift range $0.2<z<0.5$, corresponding to our EHAGNs having [Ne v] in the spectral window. (This

\footnotetext{
4 IRAF is distributed by the National Optical Astronomy Observatory, which is operated by the Association of Universities for Research in Astronomy, Inc., under cooperative agreement with the National Science Foundation.
}

Table 3

Line Ratios for Equal-height DPAGNs

\begin{tabular}{lccc}
\hline \hline SDSS Name & {$[\mathrm{O}$ III] Flux } & {$[\mathrm{Ne}$ v] Flux } & {$[\mathrm{Ne} \mathrm{v}] /[\mathrm{O}$ III $]$} \\
\hline J113105.07+610405.1 & 160 & $<18$ & $<0.110$ \\
J131018.47+250329.5 & 1419 & 80.0 & 0.056 \\
J151518.29+551535.3 & 326 & 20.3 & 0.062 \\
J153231.80+420342.7 & 1214 & 90.5 & 0.075 \\
\hline
\end{tabular}

Note. Line fluxes and ratios for eye-selected EHAGNs.

sample has 3346 objects, overwhelmingly Type 1 . To create the composite, the individual spectra were normalized to an average flux $F_{\lambda}$ of unity across the entire spectrum, and then averaged together, as described by Salviander et al. (2007).) This composite has $I(\lambda 3426) / I(\lambda 5007)=0.19 \pm 0.02$, where the error reflects uncertainty in the continuum level at [Ne v]. Thus, the group of three EHAGNs has substantially weaker [Ne v] than is typical for Type 1 AGNs in SDSS. We also examined the distribution of $[\mathrm{Ne} \mathrm{V}]$ intensities in the control sample in order to assess the likelihood of getting small values for the three EHAGNs by chance. We selected the first 100 SDSS Type 1 AGNs at $z>0.5$ for measurement of [O III] and [Ne v] with SPLOT. Of the 27 objects for which [Ne v] could reliably be measured, only 4 have $[\mathrm{Ne} \mathrm{v}] /[\mathrm{O} \mathrm{III}]$ less than 0.08 , giving a probability of less than $1 \%$ that all three EHAGNs would be as low as observed by chance.

For the EFAGNs, there are 11 objects of Type 1 excluding those that are already in the EHAGN sample. Of these, six objects allowed a reliable measurement of [Ne v]. The resulting values of $[\mathrm{Ne} \mathrm{v}] /[\mathrm{O} \mathrm{III}]$ ranged from 0.049 to 0.092 , with an average of $0.069 \pm 0.006$ (error of mean). These values are again lower than the typical value for the single-peak control sample.

We conclude that the EPAGNs do have exceptionally weak $[\mathrm{Ne} \mathrm{v}]$. This implies that the EPAGNs for the most part are not binaries. The weak [Ne v] implies that the NLR of the EPAGNs is different from normal AGNs in some way that weakens the high-ionization lines. This is consistent with a rotating ring, but other models involving NLR geometry are possible. We caution that the sample is small, but the potential utility of this approach is evident.

\section{2. [O III]/H $\beta$ in Equal-peaked Objects}

For dual AGNs, the red and blue components of the narrow lines are assumed to be due to the presence of two separate NLRs, and the line ratios in the blueshifted system would in general not be equal to those of the redshifted system. For a rotating disk model, however, a single ionizing source is responsible for illuminating both systems. Therefore, equality of line ratios in the two velocity components may provide a test of the nature of the double peaks. This idea is mentioned by $\mathrm{Xu}$ \& Komossa (2009) when considering a possible rotating disk explanation for the double-peaked object SDSS J1316+1753, and by Liu et al. (2010b) in the original presentation of their double-peaked sample.

We have performed double Gaussian fits for the $\mathrm{H} \beta$ lines in our EPAGNs and for the other DPAGNs from Smith et al. (2010) that exhibited double-peaked $\mathrm{H} \beta$. Only Type 2 objects are considered, as separation of the broad and narrow components of $\mathrm{H} \beta$ in Type 1 objects proved impractical. There are five EHAGNs and five EFAGNs usable for this purpose. From these results and the double Gaussian fits of the [O III] $\lambda 5007$ 


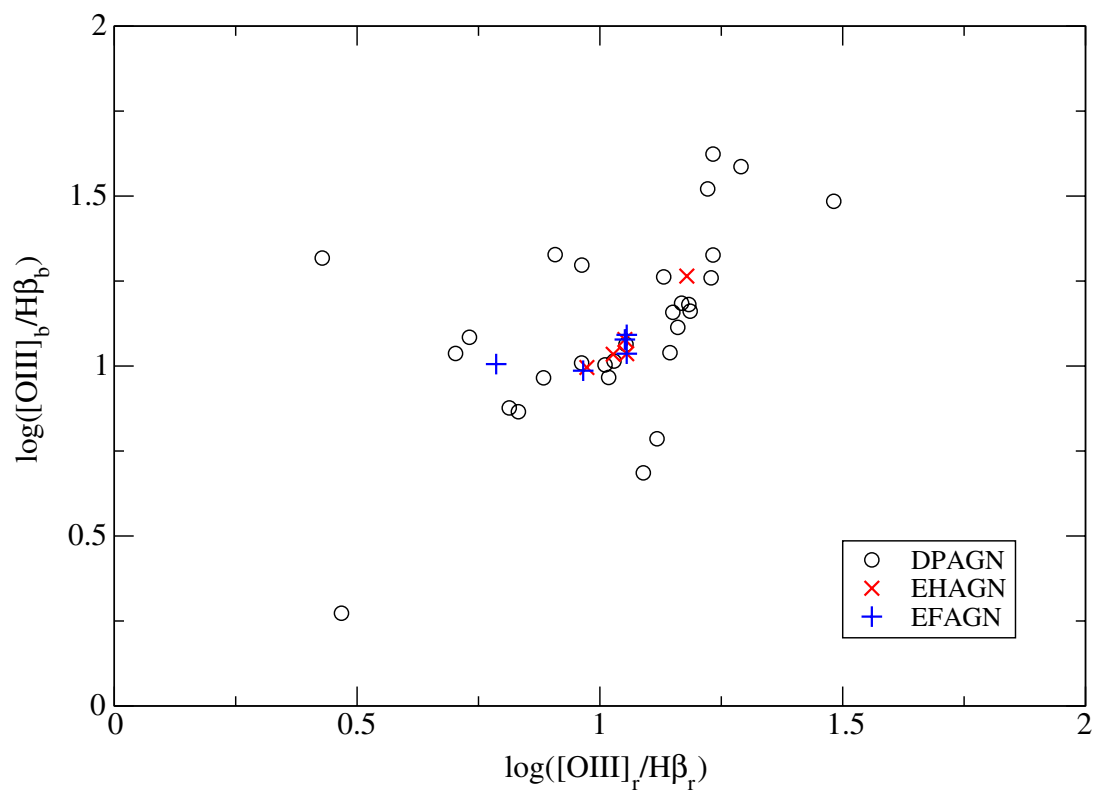

Figure 2. Ratios of the $[\mathrm{O} \mathrm{III}] / \mathrm{H} \beta$ lines for the blueshifted systems of the Type 2 DPAGN to those of the redshifted systems. A correlation exists similar to the one reported in Liu et al. (2010b). Hollow circles represent the overall DPAGNs, red crosses represent the eye-selected equal-height AGNs (EHAGNs), and blue plus signs represent the equal-flux AGNs (EFAGNs). The EPAGNs appear to occupy a tighter 1:1 correlation than the overall sample, consistent with rotating disks.

(A color version of this figure is available in the online journal.)

lines mentioned above, we calculate the $[\mathrm{O} \mathrm{III}] / \mathrm{H} \beta$ ratio for the redshifted and blueshifted systems of each object, and compare them. We create the ratio of ratios $R_{r} / R_{b}$, where $R_{r}=$ $[\mathrm{O} \mathrm{III}]_{r} / \mathrm{H} \beta_{r}$ is the line ratio for the redshifted system and likewise for the blueshifted system. As a measure of how similar these ratios are for a given object, we use the value $\left|1-R_{r} / R_{b}\right|$. For the EHAGN and the EFAGN samples, we obtain average values $\left\langle\left|1-R_{r} / R_{b}\right|\right\rangle=0.07 \pm 0.03$ and $0.13 \pm 0.09$ (error of mean), respectively.

How do these values compare with what would be expected for dual AGNs? We formed a control sample of 25 Type 2 AGNs selected by eye from the parent sample of SDSS AGNs. We measured the [O III] and $\mathrm{H} \beta$ line fluxes and calculated the $[\mathrm{O}$ III $] / \mathrm{H} \beta$ line ratios for these objects. These ratios were then randomly combined with one another in pairs to examine the distribution of values of $\left\langle\left|1-R_{r} / R_{b}\right|\right\rangle$. We obtained an average value of $\left\langle\left|1-R_{r} / R_{b}\right|\right\rangle=0.52 \pm 0.13$ (error of the mean). This exceeds the value for the EHAGNs by more than $3 \sigma$ and for the EFAGNs by more than $2 \sigma$. Thus, the line ratios of the redshifted and blueshifted systems are more similar to one another among the EPAGNs than expected for random pairings of normal quasars. This is suggestive of a single ionizing source and inconsistent with binaries.

As a further test of the statistical significance of this result, we created an independent set of 10 random pairings of the list of 25 Type 2 single-peak sample with itself, giving 10 sets of 25 simulated binaries. For each of these 10 pairings, we selected at random 72 subsets of 5 objects, giving 720 such subsets. For these subsets of five simulated objects, the average value of $\left\langle\left|1-R_{r} / R_{b}\right|\right\rangle$ was 0.51 , with an rms scatter of 0.10 . The minimum value was 0.10 , and 12 cases had $\left\langle\left|1-R_{r} / R_{b}\right|\right\rangle<0.20$. We conclude that a result of 0.07 (for the EHAGNs) or 0.12 (for the EFAGNs) will occur by chance with a probability of $\lesssim 1 \%$.

For the Type 2 DPAGN sample as a whole, excluding the equal-peaked objects, we find $\left\langle\left|1-R_{r} / R_{b}\right|\right\rangle=0.26 \pm 0.09$ (error of the mean). This value is intermediate between the EPAGNs and the control sample, but the difference is only marginally significant in terms of the error bars. In Figure 2, we plot the values of the redshifted and blueshifted systems against one another. It can be seen that these values are correlated in the same manner as in Figure 4 of Liu et al. (2010b). The Spearman correlation coefficient for our entire Type 2 DPAGN sample is $\rho=0.60$, with a probability of null correlation $P_{0}=9.52 \times 10^{-4}$. The number of EPAGNs is too small to form such a statistic. However, it appears that the EPAGNs are less scattered and lie nearer to the 1:1 line than the DPAGNs in general. The intermediate degree of correlation of $R_{r}$ with $R_{b}$ for the general DPAGN sample is consistent with a mix of binaries, disks, and jets, but such an interpretation may not be unique.

\section{CONCLUSION}

AGNs with double-peaked narrow emission lines are attracting increased attention as candidates for dual AGNs. DPAGNs offer potential insights into several aspects of AGN physics. If caused by binary AGNs, they offer a way to assess the probability of simultaneous fueling of both black holes in a galactic merger. If caused by rotating disks or radio jets, they offer insights into NLR geometry and gas dynamics. Radio, X-ray, and optical imaging, together with spatially resolved spectroscopy, are required to take advantage of these opportunities.

In this paper, we have focused on the case in which the two peaks of the [O III] line have nearly equal intensities. Such objects have weaker high-ionization lines than a control sample of single-peaked Type 1 AGNs, making them unlikely to be binary quasars. Line ratios for the redshifted and blueshifted systems of the EPAGNs are more similar to each other than those of the overall DPAGN sample, which in turn are more similar to each other than for a randomly combined control sample of single-peaked AGNs. These findings suggest a single ionizing source and are inconsistent with a binary scenario. It 
is plausible that such profiles often represent rotating disks, but further study is required to confirm this conclusion.

We thank Julie Comerford, Jenny Greene, Mark Whittle, and Bev Wills for helpful discussions. We thank an anonymous referee for many helpful suggestions that led to significant improvements in the manuscript. K.L.S. and G.A.S. gratefully acknowledge support from the University Cooperative Society of the University of Texas at Austin and the Jane and Roland Blumberg Cenntenial Professorship in Astronomy.

Funding for the Sloan Digital Sky Survey (SDSS) has been provided by the Alfred P. Sloan Foundation, the Participating Institutions, the National Aeronautics and Space Administration, the National Science Foundation, the U.S. Department of Energy, the Japanese Monbukagakusho, and the Max Planck Society. The SDSS Web site is http://www.sdss.org/. The SDSS is managed by the Astrophysical Research Consortium (ARC) for the Participating Institutions. The Participating Institutions are The University of Chicago, Fermilab, the Institute for Advanced Study, the Japan Participation Group, The Johns Hopkins University, the Korean Scientist Group, Los Alamos National Laboratory, the Max-Planck-Institute for Astronomy (MPIA), the Max-Planck-Institute for Astrophysics (MPA), New Mexico State University, University of Pittsburgh, University of Portsmouth, Princeton University, the United States Naval Observatory, and the University of Washington.

\section{REFERENCES}

Blecha, L., Loeb, A., \& Narayan, R. 2012, arXiv:1201:1904

Comerford, J. M., Gerke, B. F., Newman, J. A., et al. 2009, ApJ, 698, 956

Comerford, J. M., Pooley, D., Gerke, B. F., \& Madejksi, G. M. 2011, ApJ, 737, L19

Fu, H., Myers, A. D., Djorgovski, S. G., \& Yan, L. 2011, ApJ, 733, 103

Fu, H., Myers, A. D., Stockton, A., et al. 2012, ApJ, 745, 67

Gerke, B. F., Newman, J. A., Lotz, J., et al. 2007, ApJ, 660, L23

Liu, X., Greene, J. E., Shen, Y., \& Strauss, M. A. 2010a, ApJ, 715, 30L

Liu, X., Shen, Y., Strauss, M. A., \& Greene, J. E. 2010b, ApJ, 708, 427L

McGurk, R. C., Max, C. E., Rosario, D. J., et al. 2011, ApJ, 738, L2

Rosario, D., Shields, G. A., Taylor, G. B., Salviander, S., \& Smith, K. L. 2010, ApJ, 716, 131

Shen, Y., Liu, X., Greene, J., \& Strauss, M. 2011, ApJ, 735, 48

Salviander, S., Shields, G. A., Gebhardt, K., \& Bonning, E. W. 2007, ApJ, 662, 131

Smith, K. L., Shields, G. A., Bonning, E. W., et al. 2010, ApJ, 716, 866

Tingay, S. J., \& Wayth, R. B. 2011, AJ, 141, 174

Vanden Berk, D. E., Richards, G. T., Bauer, A., et al. 2001, AJ, 122, 549

Wang, J., Chen, Y., Hu, C., et al. 2009, ApJ, 705, L76

Whittle, M., \& Wilson, A. S. 2004, AJ, 127, 606

Xu, D., \& Komossa, S. 2009, ApJ, 705, L20

Zhou, H., Wang, T., Zhang, X., Dong, X., \& Li, C. 2004, ApJ, 604, L33 